\title{
Correlates of metabolic syndrome among young Brazilian adolescents population
}

\author{
Michel Coutinho dos Santos ${ }^{1 *} \mathbb{D}$, Ana Paula Cicci de Castro Coutinho ${ }^{1}$, Mônica de Souza Dantas ${ }^{1}$, \\ Letícia Ayran Medina Yabunaka', Dartagnan Pinto Guedes ${ }^{2}$ and Silvia Aparecida Oesterreich ${ }^{3}$
}

\begin{abstract}
Background: Findings available in literature indicate that metabolic syndrome (MetS) diagnosed in young ages tends to remain in adulthood. The aim of the study was to identify demographic, nutritional, anthropometric and behavioral correlates of MetS in a sample of adolescents from Dourados, Mato Grosso do Sul, Brazil.

Methodology: This is a cross-sectional school-based study involving 274 participants aged 12-18 years (186 girls and 88 boys). Anthropometric measurements were performed and a questionnaire with structured questions was applied for data collection. MetS was identified according to criteria proposed by the International Diabetes Federation. Data were statistically treated using bivariate analysis and hierarchical multiple regression.

Results: The proportion of adolescents identified with MetS was equivalent to $4.7 \%$ [95\% Cl (3.6-6.0)]. Multivariate analysis showed that older age $(\mathrm{OR}=1.22[1.04-1.73])$ and higher economic class ( $\mathrm{OR}=1.25[1.07-1.96])$ were significantly associated with MetS. Among behavioral factors, longer recreational screen time (OR=1.26 [1.05-1.94]) and low fruits/vegetables intake ( $\mathrm{OR}=1.49$ [1.23-2.41]) were independently associated with MetS. Likewise, excess body weight $(\mathrm{OR}=1.52[1.24-2.41])$ was significantly associated with the outcome.

Conclusion: The high proportion of adolescents with MetS and the identification of their correlates reinforce the need for early life style intervention and awareness programs in this population group.
\end{abstract}

Keywords: Cardiometabolic risk, Metabolic syndrome, Lifestyle, Health promotion, Youth

\section{Background}

Currently, regardless of sex, age, economic class and geographic region, overweight and obesity have become a global epidemic [1], which decisively contributes to the onset and development of metabolic syndrome (MetS) [2]. Findings available in literature indicate that $\mathrm{MetS}$ diagnosed in childhood and adolescence tends to remain in adulthood [3]. Therefore, the diagnosis of the possible presence of $M e t S$ at early ages, accompanied by control interventions, should have a favorable impact on the health of young people and the prevention of adverse outcomes in the future.

MetS refers to a set of cardiometabolic components that, when altered, favor the occurrence of cardiovascular events and diabetes. These components include excess

\footnotetext{
* Correspondence: michelsantos@ufgd.edu.br

'University Hospital of the Federal University of Grande Dourados, Dourados, MS, Brazil

Full list of author information is available at the end of the article
}

abdominal fat, high blood pressure, and altered triglyceride, lipoprotein, and blood glucose levels [4]. Currently, for the adult population, there is consensus regarding the cutoff points used to define the MetS components. However, it is not the case of adolescents, where the diagnostic criterion varies considerably among the different available proposals, with repercussion in clinical practice, making it difficult the comparison among studies [5].

Even considering this limitation and due to the importance and the need to diagnose the presence of MetS and correlates as early as possible, a number of studies have been carried out involving adolescents [6-12]. This is necessary because the adult prevalence is on the rise [13], and in the young population, evidence has pointed to similar phenomenon [9-11]. In addition, several attributes linked to health risk behaviors have been increasingly frequent in adolescents [14].

Therefore, the aim of the present study was to identify demographic, nutritional, anthropometric and behavioral 
correlates of MetS in a sample of adolescents from Dourados, Mato Grosso do Sul, Brazil.

\section{Methods}

In order to carry out the study, information from the database of a larger project with longitudinal characteristics (Health Education Program through Dietary and Physical Activity Interventions) was used, which includes adolescents enrolled in the 2nd cycle of elementary school (6th to 9th grades) and high-school (from 1st to 3rd grades) of four public schools randomly selected in the city of Dourados, Mato Grosso do Sul, Brazil. In this case, data were collected at the initial moment of the project. The intervention protocols were approved by the Research Ethics Committee of the Federal University of Grande Dourados (Protocol No. 1.434.947).

The inclusion of adolescents in the study was due to their desire to participate in the experiment. To that end, all students enrolled in the 2016 school year of the four selected schools, along with their parents/guardians, were contacted and informed of the nature and aims of the study. The criteria adopted to exclude some adolescents interested in participating in the study were: (a) any health problem that temporarily or permanently prevented participation in the study; (b) using any type of medication that could induce changes in the study variables; (c) undergoing any type of specific diet; and (d) pregnancy. Of the 1200 students contacted, 274 adolescents (186 girls and 88 boys) aged $12-18$ years confirmed participation in the project and signed the Free and Informed Consent Form.

The study performed anthropometric measures and components related to the identification of MetS, and a questionnaire consisting of items distributed in four sections: demographic aspects, eating habits, physical activity and sedentary behavior, was applied. The questionnaire was applied in a single moment, individually for each adolescent and in the place and time of classes. Data were collected between June and July/2016 and were carried out by a team of four researchers.

Regarding demographic aspects, in addition to sex and age, information related to ethnicity, economic class, schooling of parents/guardians, family structure and eventual work activity were collected. The family economic class was identified according to guidelines proposed by the National Association of Research Companies [15]. Information related to eating habits was obtained using semi-quantitative food frequency questionnaire from the Youth Risk Behavior Survey (YRBS) module, which was translated, adapted and validated for use in the Brazilian young population [16]. In this case, adolescents answered how often they consumed fruits/vegetables and sweetened products/soft drinks, taking as reference the week before data collection. From the intake frequency reported, the following indicators were considered: no intake; intake 1-4 days/week; intake $\geq 5$ days/week.

For the physical activity, the Physical Activity Questionnaire for Adolescents - PAQ-A was used, which was translated and validated for use in young Brazilians [17]. The PAQ-A consists of eight structured questions aimed at sizing different aspects of physical activity in the last seven days. The response options are coded using an increasing scale from 1 to 5 points, and the physical activity score was computed using the mean of scores assigned to each question. For the categorization of physical activity scores, specific cutoff points for sex and age based on the distribution of tertiles was used. Thus, the group of less active adolescents was stratified with PAQ-A scores $\leq 1$ st tertile. The group of moderately active adolescents had scores between the 1st and 2nd tertiles, and the group of the most active adolescents had scores $\geq 2$ nd tertile.

Sedentary behavior was evaluated by exposure to excessive screen time through structured issues about watching TV and using computer, video game, tablet, and smartphone in a typical week. A predefined time scale was provided for response, in which the adolescent indicated his option among four categories, ranging from "none" to "> 4 hours/day". The questions considered separately screen time equivalent to watching TV and using computer, video game, tablet and smartphone on weekdays and on weekends (Saturday and Sunday). Weighted average involving data of weekdays and weekends was used to identify screen time per day reported by students. Excessive screen time was defined by the combined use of TV and other screen devices for time $>2 \mathrm{~h}$ /day [18].

In the anthropometric field, height, body weight and waist circumference measurements were performed according to methodology described by the World Health Organization [19]. Body mass index (BMI) was calculated using the ratio between body mass expressed in kilograms and height expressed in meters squared $\left(\mathrm{kg} / \mathrm{m}^{2}\right)$. With BMI values, the anthropometric nutritional status of students was classified into four categories based on sex and age cutoff points proposed by the International Obesity Task Force (IOFT): low body weight, eutrophic, overweight and obese [20].

$M e t S$ was identified by analyzing the blood content of plasma lipids (triglycerides and HDL-C) and blood glucose, resting blood pressure (systolic and diastolic) and abdominal fat accumulation (waist circumference), according to criteria proposed by the International Diabetes Federation [21]. In this case, MetS was defined by the presence of high waist circumference $(<16$ years: both sexes $\geq$ Percentile $90, \geq 16$ years: boys $\geq 90 \mathrm{~cm}$ and girls $\geq 80 \mathrm{~cm}$ ) and at least two other compromised components: increased triglycerides ( $\geq 150 \mathrm{mg} / \mathrm{dL}$ ), low HDL-C ( $<16$ years: both sexes $<40 \mathrm{mg} / \mathrm{dL}, \geq 16$ years: boys $<40 \mathrm{mg} / \mathrm{dL}$ and girls $<50 \mathrm{mg} / \mathrm{dL}$ ), high fasting blood 
Table 1 Descriptive information of the sample selected in the study

\begin{tabular}{|c|}
\hline Demographic Indicators \\
\hline Sex \\
\hline Girls \\
\hline Boys \\
\hline Age \\
\hline $12-15$ years \\
\hline $16-18$ years \\
\hline Ethnicity \\
\hline Caucasian \\
\hline Non-Caucasian \\
\hline Economic class \\
\hline Class D-E (Low) \\
\hline Class C \\
\hline Class B-A (High) \\
\hline Schooling of Parents/Guardians \\
\hline$\leq 4$ years \\
\hline $5-8$ years \\
\hline $9-11$ years \\
\hline$\geq 12$ years \\
\hline Family structure \\
\hline Father and mother \\
\hline Separated Parents \\
\hline Relatives \\
\hline Labor Activity \\
\hline None \\
\hline Eventual \\
\hline$\geq 20 \mathrm{~h} /$ week \\
\hline Nutritional status Anthropometric \\
\hline Body mass index \\
\hline Low weight \\
\hline Normal weight \\
\hline Overweight \\
\hline Obesity \\
\hline
\end{tabular}

Behavioral indicators

Physical activity

Less active

Moderately Active

More active

Screen Time

$\leq 2 \mathrm{~h} /$ day

$>2$ h/day

Fruits/vegetables intake

No intake

Intake 1-4 days/week n (\%)

$186(67.9)$

88 (32.1)

$159(58.0)$

$115(42.0)$

$199(72.6)$

75 (27.4)

99 (36.1)

$107(39.1)$

$68(24.8)$

$82(29.9)$

59 (21.5)

55 (20.1)

78 (28.5)

$182(66.4)$

64 (23.4)

$28(10.2)$

209 (76.3)

$20(7.3)$

45 (16.4)

$18(6.6)$

$185(67.5)$

42 (15.3)

29 (10.6)

$136(49.7)$

$76(27.7)$

$62(22.6)$

66 (24.1)

$208(75.9)$

44 (16.1)

$156(56.9)$
Table 1 Descriptive information of the sample selected in the study (Continued)

\begin{tabular}{ll}
\hline & $\mathrm{n}(\%)$ \\
\hline Intake $\geq 5$ days/week & $74(27.0)$ \\
No intake & $18(6.6)$ \\
Intake 1-4 days/week & $126(46.0)$ \\
Intake $\geq 5$ days/week & $130(47.4)$ \\
Metabolic Syndrome Components & \\
High Waist Circumference & $42(15.3)$ \\
Increased Triglyceride & $18(6.6)$ \\
Decreased HDL-cholesterol & $69(25.2)$ \\
Elevated fasting blood glucose & $14(5.1)$ \\
Altered Blood Pressure & $24(8.8)$ \\
\hline
\end{tabular}

glucose $(\geq 100 \mathrm{mg} / \mathrm{dL})$ and altered blood pressure (systolic $\geq 130 \mathrm{mmHg}$ or diastolic $\geq 85 \mathrm{mmHg}$ ).

Data were statistically treated using the Statistical Package for Social Science (SPSS), version 22. The observed proportions (\%) in the outcome of Interest (MetS) according to demographic, anthropometric, behavioral and nutritional correlates were presented with respective 95\% confidence intervals (95\% CI). To analyze the linearity of associations between $M e t S$ and potential correlates, prevalence ratio calculations were used. Statistical differences among strata under investigation were treated by the chi-square test $\left(x^{2}\right)$. In the sequence, correlates that indicated at least marginally significant associations $(p \leq 0.20)$ in the bivariate analysis were selected to be included in hierarchical multiple regression procedures. In this case, correlates were included in blocks, and demographic aspects (level one) were the first to be included the model, followed by the anthropometric nutritional indicator (level two) and, finally, the behavioral components were included (level three). All correlates that presented statistical significance remained in the multivariate model, $p<0.05$.

\section{Results}

Descriptive information characterizing the sample selected for the study is provided in Table 1 . Approximately $1 / 3$ of the sample is composed of boys (32.1\%) and the highest concentration of adolescents is aged 12-15 years (58\%). Most adolescents in the study were Caucasian (72.6\%), live with parents (66.4\%) and did not work (76.3\%). Regarding the economic class and schooling of parents/guardians, the adolescents were proportionally distributed in the strata considered. In addition, 25.9\% of adolescents were overweight (overweight + obesity), almost half were classified as minimally physically active (49.7\%), and in each group of ten adolescents, seven reported remaining over $2 \mathrm{~h}$ in screen devices. Daily fruit/vegetable intake was reported by $27 \%$ of adolescents and $93.4 \%$ of them assumed 
Table 2 Prevalence $(95 \% \mathrm{Cl}$ ) and prevalence ratio (95\% Cl) of metabolic syndrome with stratification for demographic, nutritional and behavioral correlates of adolescents from Dourados, Mato Grosso do Sul, Brazil, 2016

\begin{tabular}{|c|c|c|c|c|}
\hline & & Prevalence $(95 \% \mathrm{Cl})$ & Prevalence Ratio & $p$-value \\
\hline & Overall & $4.7(3.6-6.0)$ & & \\
\hline \multicolumn{5}{|l|}{ Demographic Indicators } \\
\hline \multirow[t]{3}{*}{ Sex } & & & & 0.217 \\
\hline & Girls & $4.4(3.5-5.4)$ & Reference & \\
\hline & Boys & $5.0(3.8-6.4)$ & $1.10(0.95-1.41)$ & \\
\hline \multirow[t]{3}{*}{ Age } & & & & 0.173 \\
\hline & $12-15$ years & $4.2(3.2-5.3)$ & Reference & \\
\hline & $16-18$ years & $5.2(4.0-6.6)$ & $1.21(1.03-1.63)$ & \\
\hline \multirow[t]{3}{*}{ Ethnicity } & & & & 0.341 \\
\hline & Caucasian & $4.9(3.8-6.1)$ & $1.04(0.92-1.29)$ & \\
\hline & Non-Caucasian & $4.6(3.5-5.8)$ & Reference & \\
\hline \multirow[t]{4}{*}{ Economic class } & & & & 0.139 \\
\hline & Class D-E (Low) & $4.3(3.3-5.4)$ & Reference & \\
\hline & Class C & $4.6(3.6-5.8)$ & $1.05(0.93-1.39)$ & \\
\hline & Class B-A (High) & $5.4(4.2-6.8)$ & $1.23(1.04-1.68)$ & \\
\hline \multirow[t]{5}{*}{ Schooling of Parents/Guardians } & & & & 0.166 \\
\hline & $\leq 4$ years & $4.2(3.3-5.1)$ & Reference & \\
\hline & $5-8$ years & $4.6(3.5-5.9)$ & $1.07(0.94-1.34)$ & \\
\hline & $9-11$ years & $4.9(3.7-6.4)$ & $1.14(0.98-1.46)$ & \\
\hline & $\geq 12$ years & $5.2(3.9-6.7)$ & $1.21(1.01-1.67)$ & \\
\hline \multirow[t]{4}{*}{ Family structure } & & & & 0.367 \\
\hline & Father and mother & $4.9(3.8-6.1)$ & $1.04(0.93-1.30)$ & \\
\hline & Separated Parents & $4.6(3.5-5.8)$ & Reference & \\
\hline & Relatives & $4.7(3.7-5.8)$ & $1.00(0.89-1.36)$ & \\
\hline \multirow[t]{4}{*}{ Labor Activity } & & & & 0.274 \\
\hline & None & $4.9(3.8-6.1)$ & $1.03(0.94-1.30)$ & \\
\hline & Eventual & $4.6(3.4-5.9)$ & Reference & \\
\hline & $\geq 20 \mathrm{~h} /$ week & $5.1(3.9-6.5)$ & $1.08(0.95-1.35)$ & \\
\hline \multicolumn{5}{|l|}{ Nutritional Anthropometric status } \\
\hline \multirow[t]{5}{*}{ Body mass index } & & & & $<0.001$ \\
\hline & Low weight & $4.0(3.4-4.7)$ & Reference & \\
\hline & Normal weight & $4.4(3.5-5.5)$ & $1.08(0.96-1.33)$ & \\
\hline & Overweight & $4.9(3.8-6.1)$ & $1.21(1.02-1.69)$ & \\
\hline & Obesity & $5.6(4.4-6.8)$ & $1.38(1.15-1.90)$ & \\
\hline \multicolumn{5}{|l|}{ Behavioral indicators } \\
\hline \multirow[t]{4}{*}{ Physical activity } & & & & 0.181 \\
\hline & Less active & $5.1(4.0-6.3)$ & $1.19(1.01-1.67)$ & \\
\hline & Moderately Active & $4.8(3.8-5.9)$ & $1.12(0.96-1.45)$ & \\
\hline & More active & $4.2(3.4-5.2)$ & Reference & \\
\hline \multirow[t]{3}{*}{ Screen Time } & & & & 0.119 \\
\hline & $\leq 2 \mathrm{~h} /$ day & $4.2(3.3-5.3)$ & Reference & \\
\hline & $>2$ h/day & $5.3(4.1-6.7)$ & $1.24(1.02-1.69)$ & \\
\hline
\end{tabular}


Table 2 Prevalence $(95 \% \mathrm{Cl}$ ) and prevalence ratio (95\% Cl) of metabolic syndrome with stratification for demographic, nutritional and behavioral correlates of adolescents from Dourados, Mato Grosso do Sul, Brazil, 2016 (Continued)

\begin{tabular}{llll}
\hline & & Prevalence $(95 \% \mathrm{Cl})$ & Prevalence Ratio \\
\hline Fruits/vegetables intake & No consumption & $5.4(4.2-6.8)$ & $1.35(1.13-1.91)$ \\
& Intake 1-4 days/week & $4.9(4.8-6.1)$ & $1.22(1.03-1.73)$ \\
& Intake $\geq 5$ days/week & $3.9(3.2-4.8)$ & Reference \\
Sweetened products/soft drinks intake & & & Reference \\
& No intake & $4.3(3.3-5.4)$ & $1.04(0.94-1.34)$ \\
& Intake 1-4 days/week & $4.6(3.5-5.9)$ & $1.20(1.01-1.69)$ \\
\hline
\end{tabular}

to consume sweetened products/soft drinks at least once a week. Regarding the individual components of MetS, decreased HDL-cholesterol was predominant (25.2\%), while increased triglyceride (6.6\%) and high fasting blood glucose (5.1\%) were the least prevalent.

The presence of MetS with stratification for demographic, nutritional status and behavioral correlates are presented in Table 2. Overall prevalence was equivalent to $4.7 \%$ [95\% CI (3.6-6.0)]. Results from bivariate analyses showed that, from the list of potential correlates considered, sex, ethnicity, family structure and labor activity were not statistically indicated $(p<0.20)$.

Results of the hierarchical multiple regression are available in Table 3. In the case of demographic correlates, the final model pointed to significant associations between $M e t S$, age and economic class. Likewise, the anthropometric correlate related to nutritional status (BMI) remained significantly associated with MetS. Among behavioral components, of the four correlates considered, two of them (screen time and fruits/vegetables intake) remained associated with the outcome.

\section{Discussion}

The aim of the study was to provide up-to-date information on demographic, anthropometric and behavioral nutritional correlates of $M e t S$ in a sample of adolescents from Dourados, Mato Grosso do Sul, Brazil. The main findings were that, using the same diagnostic criteria (IDF), the proportion of adolescents with MetS was higher than that found in the young Brazilian population [4.5\% vs. 2.6\%] [12]. When compared with international data, the proportion observed in the present study is lower than that reported in North American and European adolescents; however, higher than that found in adolescents from Asian countries [9-11]. Also, in addition to being older and belonging to higher economic class, adolescents identified with $M e t S$ were those who reported excessive screen time, lower fruits/vegetables intake and greater body weight accumulation.
In general, correlates of MetS identified in the present study are consistent with current literature [6-8]. However, it is noteworthy that, unlike the findings of some studies [9, 10]; but consistent with others [11, 12], sex had no significant association with the presence of MetS. In this case, possibly the differences observed among studies can be attributed to the several criteria used to identify MetS in children and adolescents, since to date, there is no consensus regarding the use of a single criterion [5]. Another hypothesis to be considered may be related to the known differences between sexes related to the prevalence of abdominal obesity, hypertension and dyslipidemia found in different young populations [9]. in more advanced ages.

Corroborating previous findings [6-12], age was a significant correlate for the presence of MetS among adolescents in this study. In this regard, the expected increase in blood pressure, triglyceride and fasting blood glucose levels, and visceral fat deposits in more advanced ages [22] may explain the higher proportion of MetS among older adolescents. Regarding the economic class, the presence of MetS was positively associated with the higher strata. Other studies have identified significant associations in the same direction $[6,8]$; however, we also find information in literature pointing to an inverse relationship between economic class and presence of MetS [12]. Perhaps the different indicators used to classify the economic class and the interactions between family income and attributes of the socio-cultural context in which it is inserted can contribute to understand these differences.

Regarding sedentary behavior, in a certain way, the results found here coincides with results found in other studies and suggest that the possibility of the presence of $M e t S$ is progressively higher according to the increase in screen time reported by adolescents $[8,23,24]$. However, a fact that should be considered refers to the cutoff point used to define excessive screen time, typically recommended by current international guidelines and used in the present study ( $>2 \mathrm{~h}$ /day). In this sense, in a study involving meta-analysis resources, primary and sensitivity 
Table 3 Hierarchical multiple logistic regression for demographic (level 1), nutritional (level 2) and behavioral (level 3) correlates of metabolic syndrome of adolescents from Dourados, Mato Grosso do Sul, Brazil, 2016

\begin{tabular}{|c|c|c|}
\hline Correlates & OR crude $(95 \% \mathrm{Cl})^{\mathrm{a}}$ & $\mathrm{OR}_{\text {Adjusted }}(95 \% \mathrm{Cl})^{\mathrm{b}}$ \\
\hline \multicolumn{3}{|c|}{ Level 1 - Demographic Indicators } \\
\hline \multicolumn{3}{|l|}{ Age } \\
\hline $12-15$ years & Reference & Reference \\
\hline $16-18$ years & $1.26(1.03-1.88)$ & $1.22(1.04-1.73)$ \\
\hline \multicolumn{3}{|l|}{ Economic class } \\
\hline Class D-E (Low) & Reference & Reference \\
\hline Class C & $1.17(0.98-1.73)$ & $1.15(0.97-1.61)$ \\
\hline Class B-A (High) & $1.29(1.03-2.13)$ & $1.25(1.07-1.96)$ \\
\hline \multicolumn{3}{|c|}{ Schooling of Parents/Guardians } \\
\hline$\leq 4$ years & Reference & Reference \\
\hline $5-8$ years & $1.14(0.95-1.77)$ & $1.11(0.93-1.71)$ \\
\hline $9-11$ years & $1.18(0.97-1.95)$ & $1.14(0.96-1.82)$ \\
\hline$\geq 12$ years & $1.24(1.01-2.06)$ & $1.19(0.98-1.89)$ \\
\hline \multicolumn{3}{|c|}{ Level 2 - Nutritional Anthropometric status } \\
\hline \multicolumn{3}{|l|}{ Body mass index } \\
\hline Low weight & Reference & Reference \\
\hline Normal weight & $1.16(0.97-1.77)$ & $1.10(0.95-1.69)$ \\
\hline Overweight & $1.25(1.02-2.17)$ & $1.18(0.98-1.99)$ \\
\hline Obesity & $1.67(1.29-2.78)$ & $1.52(1.24-2.41)$ \\
\hline \multicolumn{3}{|l|}{ Level 3 - Behavioral indicators } \\
\hline \multicolumn{3}{|l|}{ Physical activity } \\
\hline Less active & $1.23(1.02-2.04)$ & $1.18(0.98-1.87)$ \\
\hline Moderately Active & $1.19(0.99-1.83)$ & $1.13(0.96-1.69)$ \\
\hline More active & Reference & Reference \\
\hline \multicolumn{3}{|l|}{ Screen Time } \\
\hline$\leq 2$ h/day & Reference & Reference \\
\hline$>2$ h/day & $1.29(1.05-2.11)$ & $1.26(1.05-1.94)$ \\
\hline \multicolumn{3}{|l|}{ Fruits/vegetables intake } \\
\hline No intake & $1.59(1.24-2.65)$ & $1.49(1.23-2.41)$ \\
\hline Intake 1-4 days/week & $1.29(1.08-2.27)$ & $1.24(1.06-2.03)$ \\
\hline Intake $\geq 5$ days/week & Reference & Reference \\
\hline \multicolumn{3}{|c|}{ Sweetened products/soft drinks intake } \\
\hline No intake & Reference & Reference \\
\hline Intake 1-4 days/week & $1.15(0.94-1.98)$ & $1.09(0.93-1.76)$ \\
\hline Intake $\geq 5$ days/week & $1.21(1.01-2.17)$ & $1.14(0.97-1.96)$ \\
\hline
\end{tabular}

annadjusted odds ratio

${ }^{b}$ Odds ratio adjusted by the other variables included in the model

analyses performed based on this cutoff point did not reveal significant associations between screen time and MetS. In this case, individual studies included in the meta-analysis that indicated significant associations adopted cutoff points close to $4 \mathrm{~h}$ /day [25].
Regarding physical activity, no significant associations between scores equivalent to PAQ-A and MetS were found. To our knowledge, this was the first study that used PAQ-A to address possible associations between physical activity and MetS. However, according to current literature, it was verified that studies involving other types of questionnaires also found no significant differences in the physical activity of adolescents identified and not with MetS [26, 27]. On the other hand, studies that identified significant association between low levels of physical activity and greater chance of identifying $M e t S$ used the accelerometry technique [28] as a measure of physical activity. These findings are confirmed through meta-analysis resources, which demonstrated the reliance on the use of accelerometers to identify significant associations between physical activity practice and MetS in young populations [25].

Another finding from the present study was the significant association detected between fruits/vegetables intake and MetS. It is important to highlight that this food habit remained significantly associated even through adjustments to potential confounding variables. In this case, the protection attributed to higher fruits/vegetables intake is consistent with evidence presented by other studies involving different experimental designs and statistical treatments $[6-8,26]$. Dietary pattern exerts influence on MetS through a specific effect on the plasma lipid-lipoprotein profile, blood pressure and body fat. Unlike diets in which fat-rich foods predominate, diets with higher fruits/vegetables intake tend to have lower intake of simple carbohydrates and saturated fat, while higher amounts of complex carbohydrates and fibers are inversely related to altered blood glucose and triglycerides, increased accumulation of abdominal fat and high blood pressure, and positively with more favorable HDL-C, all known components of MetS [29].

Based on the findings of the present study, the higher the excess body weight, the higher the proportion of $M e t S$, with an abrupt increase in obesity cases. Similar findings are found in literature $[6-9,12]$, which corroborates the hypothesis that anthropometric nutritional status is strongly associated with MetS. In this sense, studies have detected important associations between obesity and MetS from early ages. Using a longitudinal design, among a set of biological and behavioral variables, it was found that childhood obesity is the strongest predictor of MetS and other risk factors for cardiovascular diseases in early adulthood [30].

The research method used to identify behavioral indicators involved a self-report questionnaire, thus allowing possible memory bias or even tendentious statements towards the desirable, being among the study limitations. However, the reporting of these indicators by adolescents themselves is a current procedure in studies with 
these characteristics, being the most feasible form of school-based or population-based surveys. In addition, the small sample size may somehow potentiate possible inaccuracy of the calculated estimates. The cross-sectional approach of data does not allow making inferences of causality in the association between the identification of MetS and the investigated correlates, because the outcome and the other variables have been identified at the same time.

\section{Conclusions}

In conclusion, the results of the study are considered as a preliminary observation among the young Brazilian adults population as a high risk group for MetS. In approximately $5 \%$ of adolescents selected in this study the presence of MetS was identified, with emphasis on older individuals and those of higher economic class. Significant inverse associations between MetS and fruits/vegetables intake, coupled with the direct association with higher screen time and greater body weight accumulation, suggest that policies and interventions aimed at health education programs targeting school and family contexts should include actions focusing on the attempt to reduce the incidence of $M e t S$.

\section{Abbreviations}

BMI: Body mass index; HDLc: High density lipoproteins; IC95\%: 95\% Confidence intervals; IDF: International diabetes federation; IOFT: International obesity task force; MetS: Metabolic syndrome; PAQA: Physical activity questionnaire for adolescents; SPSS: Statistical package for the social science; YRBS: Youth risk behavior survey; X2: Chi-square test

\section{Acknowledgments}

We thank all of the data collection: José Botelho Sena Neto, Nerilda Ribeira Veiga, Adriana Favero de Lima, Mara Lourenço Vermeiro, Mi Ye Marcaida Olimpio, Luis Henrique Almeida Castro and the clinical analysis team: Viviane Regina Noro and Andessa Leite Ferraz de Melo.

\section{Funding}

The study was partially funded by the Federal University of Grande Dourados and the University Hospital of Grande Dourados.

\section{Availability of data and materials}

Data supporting the results of this study are available from [name of third party], but the restrictions apply to the availability of this data, which was used under license for the current study and therefore not publicly available. The data are, however, available by the authors upon reasonable request and with permission of [name of third party]

\section{Authors' contributions}

MCS acquisition of data and been involved in drafting the manuscript, APCCC acquisition of data, revising it critical, MSD acquisition of data, and been involved in drafting the manuscript, LAMY acquisition of data and involved in the laboratory analysis of biological samples, DPG contributions to conception and design, statistical analysis and corrected the manuscript, SAO contributions to conception and design, analysis and interpretation of data, general supervision of the research. All authors read and approved the final manuscript.

\section{Ethics approval and consent to participate}

The intervention protocols were approved by the Research Ethics Committee of the Federal University of Grande Dourados (Protocol No. 1.434.947).

\section{Competing interests}

The authors declare that they have no competing interests in this section.

\section{Publisher's Note}

Springer Nature remains neutral with regard to jurisdictional claims in published maps and institutional affiliations.

\section{Author details}

${ }^{1}$ University Hospital of the Federal University of Grande Dourados, Dourados, MS, Brazil. ${ }^{2}$ Northern University of Paraná, Londrina, PR, Brazil. ${ }^{3}$ Faculty of Health Sciences, Federal University of Grande Dourados, Dourados, MS, Brazil.

Received: 13 March 2018 Accepted: 20 June 2018

Published online: 06 July 2018

\section{References}

1. Ng M, Fleming T, Robinson M, Thonsom B, Graetz N. Margono, et al. global, regional, and national prevalence of overweight and obesity in children and adults during 1980-2013: a systematic analysis for the global burden of disease study 2013. Lancet. 2014;384(9945):766-81.

2. Zimmet P, Alberti G, Kaufman F, Tajima N, Silink M, Arslanian S, et al. The metabolic syndrome in children and adolescents. Lancet. 2007;369(9579): 2059-61.

3. Magnussen CG, Koskinen J, Chen W, Thomson R, Schmidt MD, Srinivasan SR, et al. Pediatric metabolic syndrome predicts adulthood metabolic syndrome, subclinical atherosclerosis, and type 2 diabetes mellitus but is no better than body mass index alone: the Bogalusa heart study and the cardiovascular risk in young Finns study. Circulation. 2010;122(16):1604-11.

4. Alberti KG, Eckel RH, Grundy SM, Zimmet PZ, Cleeman Jl, Donato KA, et al. Harmonizing the metabolic syndrome: a joint interim statement of the international diabetes federation task force on epidemiology and prevention; National Heart, Lung, and Blood Institute; American Heart Association; world heart federation; international atherosclerosis society; and International Association for the Study of obesity. Circulation. 2009;120(16):1640-5.

5. Agudelo GM, Bedoya G, Estrada A, Patiño FA, Muñoz AM, Velásquez CM. Variations in the prevalence of metabolic syndrome in adolescents according to different criteria used for diagnosis: which definition should be chosen for this age group? Metab Syndr Relat Disord. 2014;12(4):202-9.

6. Ekelund U, Anderssen S, Andersen LB, Riddoch CJ, Sardinha LB, Luan J, et al. Prevalence and correlates of the metabolic syndrome in a population-based sample of European youth. Am J Clin Nutr. 2009;89(1):90-6.

7. You MA, Son YJ. Prevalence of metabolic syndrome and associated risk factors among Korean adolescents: analysis from the Korean national survey. Asia Pac J Public Health. 2012;24(3):464-71.

8. Mehairi AE, Khouri AA, Naqbi MM, Muhairi SJ, Maskari FA, Nagelkerke N, et al. Metabolic syndrome among Emirati adolescents: a school-based study. PLoS One. 2013;8(2):e56159.

9. Friend A, Craig L, Turner S. The prevalence of metabolic syndrome in children: a systematic review of the literature. Metab Syndr Relat Disord. 2013;11(2):71-80.

10. Tailor AM, Peeters PH, Norat T, Vineis P, Romaquera D. An update on the prevalence of the metabolic syndrome in children and adolescents. Int J Pediatr Obes. 2010:5(3):202-13.

11. Poyrazoglu S, Bas F, Darendeliler F. Metabolic syndrome in young people. Curr Opin Endocrinol Diabetes Obes. 2014;21(1):56-63.

12. Kuschnir MC, Bloch KV, Szklo M, Klein CH, Barufaldi LA, Abreu GA, et al. ERICA: prevalence of metabolic syndrome in Brazilian adolescents. Rev Saúde Pública. 2016;50(supl 1):11s.

13. Grundy SM. Metabolic syndrome pandemic. Arterioscler Thromb Vasc Biol. 2008;28(4):629-36.

14. Nunes HEG, Gonçalves ECA, Vieira JAJ, Silva DAS. Clustering of risk factors for non-communicable diseases among adolescents from southern Brazil. PLoS One. 2016;11(7):e0159037.

15. Associação Brasileira de Empresas de Pesquisa - ABEP. Critério de Classificação Econômica Brasil. São Paulo: Associação Brasileira de Empresas de Pesquisa; 2014.

16. Guedes DP, Lopes CC. Validation of the Brazilian version of the 2007 youth risk behavior survey. Rev Saude Publica. 2010;44(5):840-50.

17. Guedes DP. Measuring physical activity in Brazilian youth: reproducibility and validity of the PAQ-C and PAQ-A. Rev Bras Med Esporte. 2015;21(6):425-32.

18. American Academy of Pediatrics; Council on Communications and Media. Children, adolescents, obesity, and the media. Pedriatrics. 2011;128(1):201-8. 
19. WHO. World Health Organization. Physical status: the use and interpretation of anthropometry. Report of a WHO expert committee. [technical report series nْ 854]. Geneva: WHO; 1995.

20. Cole TJ, Lobstein T. Extended international (IOTF) body mass index cut-offs for thinness, overweight and obesity. Pediatr Obes. 2012;7(4):284-94.

21. Zimmet P, Alberti KG, Kaufman F, Tajima N, Silink M, Arslanian S, et al. The metabolic syndrome in children and adolescents - an IDF consensus report. Pediatr Diabetes. 2007;8(5):299-306.

22. Werneck AO, Silva DR, Collings PJ, Fernandes RA, Ronque ERV, Barbosa DS, et al. Biological maturation, central adiposity, and metabolic risk in adolescents: a mediation analysis. Child Obes. 2016;12(5):377-83.

23. Kang HT, Lee HR, Shim JY, Shin YH, Park BJ, Lee YJ. Association between screen time and metabolic syndrome in children and adolescents in Korea: the 2005 Korean National Health and nutrition examination survey. Diabetes Res Clin Pract. 2010;89(1):72-8.

24. Mark AE, Janssen I. Relationship between screen time and metabolic syndrome in adolescents. J Public Health. 2008;30(2):153-60.

25. Oliveira RG, Guedes DP. Physical activity, sedentary behavior, cardiorespiratory fitness and metabolic syndrome in adolescents: systematic review and metaanalysis of observational evidence. PLoS One. 2016;11(12):e0168503.

26. Pan Y, Pratt CA. Metabolic syndrome and its association with diet and physical activity in US adolescents. J Am Diet Assoc. 2008;108(2):276-86.

27. Budak N, Oztürk A, Mazicioglu M, Yazici C, Bayram F, Kurtoglu S. Decreased high-density lipoprotein cholesterol and insulin resistance were the most common criteria in 12- to 19-year-old adolescents. Eur J Nutr. 2010;49(4): 219-25

28. Nguyen TH, Tang HK, Kelly P, Van d PHP, Dibley MJ. Association between physical activity and metabolic syndrome: a cross sectional survey in adolescents in ho chi Minh City, Vietnam. BMC Public Health. 2010;10:141.

29. Calton EK, James AP, Pannu PK, Soares MJ. Certain dietary patterns are beneficial for the metabolic syndrome: reviewing the evidence. Nutr Res. 2014;34(7):559-68.

30. Srinivasan SR, Myers L, Berenson GS. Predictability of childhood adiposity and insulin for developing insulin resistance syndrome (syndrome $X$ ) in young adulthood: the Bogalusa heart study. Diabetes. 2002;51(1):204-9.

\section{Ready to submit your research? Choose BMC and benefit from}

- fast, convenient online submission

- thorough peer review by experienced researchers in your field

- rapid publication on acceptance

- support for research data, including large and complex data types

- gold Open Access which fosters wider collaboration and increased citations - maximum visibility for your research: over $100 \mathrm{M}$ website views per year

At BMC, research is always in progress.

Learn more biomedcentral.com/submissions 